\title{
The Analysis Method about Change Region of Process Model based on Module
}

\author{
Xianwen Fang ${ }^{1}$, Lu Liu ${ }^{2}$ and Xiangwei Liu ${ }^{2, *}$ \\ ${ }^{1}$ Department of Information and Computing Science, Anhui University of Science and Technology, Huainan 232001, China \\ ${ }^{2}$ Department of Management, Anhui University of Science and Technology, Huainan 232007, China
}

Received: 8 Jun. 2013, Revised: 11 Oct. 2013, Accepted: 12 Oct. 2013

Published online: 1 Jan. 2014

\begin{abstract}
Business process management is a common approach of analyzing evolution and formulation of process models. But it is inevitable to come about the change problem of process models in BPMN, so that quickly and accurately seeking the change region of process models is the key thing of BPMN. Existing research is mainly dependent on the target model, and seeks the change region of process models by fixing the observation points. However, on the one hand, this way needs to compare with the target model. On the other hand, by static analysis, it can not quickly and accurately seek the change region of process models. In this paper, firstly, module decomposition and behavioural relation of module are analyzed based on Petri net and behavioural profile, without building the target model, thus avoid the inaccuracy of seeking the change region by comparing the models. Secondly, by dynamic search, this paper presents a novel way to seek the change region of process model and confirm the minimal change region of process model. Finally, this method is valid by theory and example analysis.
\end{abstract}

Keywords: Petri net, change region, module decomposition, modular behavioural profile

\section{Introduction}

With the improvement and development of scientific technology, the field of business process is increasingly wide and analytic requirements of business process model are more and more high. Different purposes of process modeling can produce different business process model, even in the same business environment. In these process models, some can well depict and complete the purpose of modeling, but some will go wrong. Therefore, to solve the change problem of process model is the key thing in process modeling.

At present, the main methods to solve the change problem of process model are analysis linguistics and behavior of the process, comparing process consistency or similarity between process models, establishing constraint model and language, etc. Based on trace equivalence, [1] presented an equivalent standard to compare syntax and semantics between process models for seeking difference. Then, to manage changes between utilized synchronous theory based on aligned differently abstract levels of process models, [2] process model. But its practical universality needed to be further improved. On the basis of the behavior profile, $[3,4]$ fixed a change point of process model to seek the change region of its matching process model by the behavioural standard of matching behavioural pairs. But to look for the accurate change region was not considered. In UML, [5] depicted that using process profile to fix the starting observation and considering inconsistent dependence, confirmed the difference. But this method had limitation. On this basis, [6] has not only better improved the method in [5], but also has defined constraint standards of consistency based on objective constraint language (OCL) and unified modeling language (UML). At the same time, it presented a new way that was agent-oriented to adjust change. In [7], firstly, it introduced functional models and physical models. Then it analyzed changes between parts based on matrix. Finally, to seek design conflicts in the design stage depended on the spread of these changes. For identifying mixed changes, [8] used EPC semantics to conclude eight types of changes who would occur. The above researches mainly compare the certain target model with it matching process model to find the change region. In confirming the change region, they mainly choose the fixed observing point to seek the change region or the

\footnotetext{
*Corresponding author e-mail: 1xw7710@ tom.com
} 
suspected change point. The results obtained in these static studies have blindness and uncertainty.

According to existing researches, this paper introduces a novel method that is behavioural analysis of module, to confirm more accurate change region in process model. Behavioural analysis of module is that performs modular decomposition of process model itself and analyzes the relationships between modules to determine change region. So it gets ride of obtain inaccurate change region, comparing with the complex and uncertain target model. In addition, this way can get accurate change region by dynamic analysis and observing different nodes, improving the disadvantage of prior mentioned static study. First of all, in process modeling, the motivative example shows the importance of seeking change region, when change in activities is uncertain. Then, to solve this problem presents modular decomposition of process model based on the definition of module and behavioural profile. Finally, to obtain more accurate change region, it analyzes change modules according to the behavioural relationship between modules, based on dynamic search and observing all output transitions of modules in process model.

The remainder of this paper is structured as follows: Section 2 introduces a motivate example. Section 3 gives some elementary notion. In section 4 , a novel way to seek change region is presented and the motivate example is analyzed. Conclusion of this paper is in section 5 .

\section{Motivative Example}

Example: in an online shopping mall, a customer discovers his receiving address is wrong after the order submitted successfully and payment finished. So he modifies the order. However, the system requires the customer to cancel the original order in the beginning, then renewedly finishing the order. In this operation, it often comes about this case: when the customer chooses payment again, the system informs the customer of non-payment (because the price is unchanged in renewed operation, the system should go all right). After analysis, there exist problems between refundment of the old order and payment of the new order. Here analyzes the system based on Petri net and Fig. 1 is the part Petri net model of the customer shopping in the online mall.

After analyzing the Fig. 1, the Petri net model of this shopping system is correctness, and can be terminated. But it will come about unexpected problems in actual implementation. It needs to solve whether there exists the problem of change region in Petri net model.

Based on existing research, the method to solve this problem in [3] has two steps: firstly, the target model is built and markets their matching transitions; secondly, a change transition as the observing point is added to the target model, and then gets the suspected change region in the dashed area of Fig. 2. However, it is inaccurate according to the dashed are of Fig. 1. To solve this

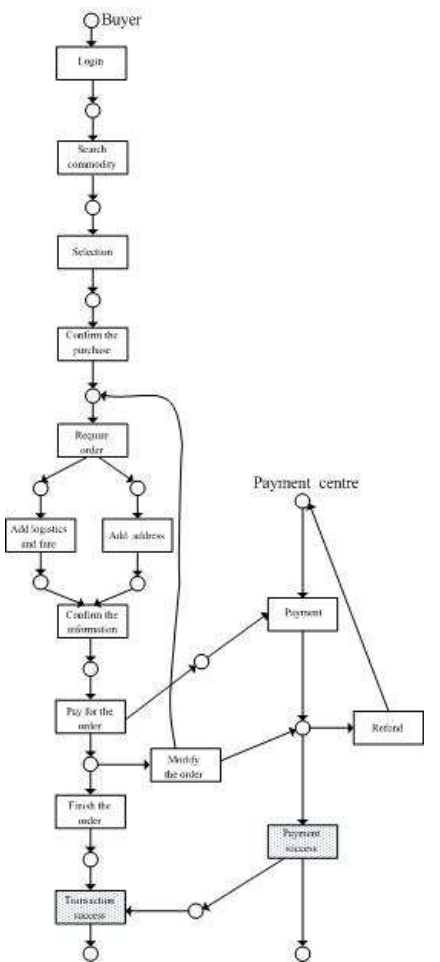

Fig. 1: Petri net model of the customer shopping in the online mall

problem, it should insert a transition who can inform the customer of successful payment between "Transaction success" and "Payment success". Consequently, to identify accurately the change region of process model, this paper puts forward a new method without target model. Especially, seeking the change region of process model uses the dynamic study.

\section{Basic Definitions}

This section focuses on the concept related to this paper closely, and other concepts see [9].

Definition 1(Petri net). A tuple $N=(S, T ; F)$ is a Petri net or a net, if

$$
\begin{aligned}
& \text { 1.S } S \cup T \neq \emptyset \\
& \text { 2. } S \cap T \neq \emptyset \\
& 3 . F \subseteq((S \times T) \cup(T \times S)) \\
& \text { 4. } \operatorname{dom}(F) \cup \operatorname{cod}(F)=S \cup T \text { and } \\
& \operatorname{dom}(F)=\{x \in S \cup T \mid \exists y \in S \cup T:(x, y) \in F\} \\
& \operatorname{cod}(F)=\{x \in S \cup T \mid \exists y \in S \cup T:(y, x) \in F\}
\end{aligned}
$$

Petri net can clearly and accurately depict the process of business process model, with its related notions in [10]. In order to understand the behavioural relationship between nodes in process, here introduces behavioural profile as follows. 


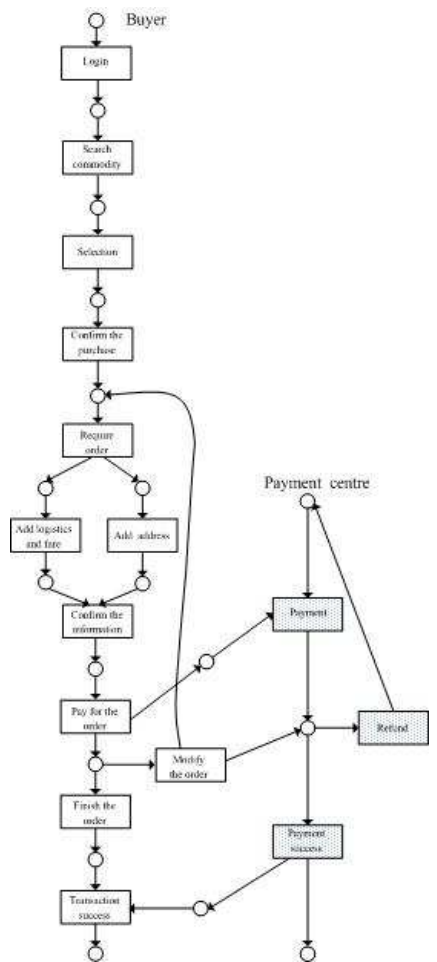

Fig. 2: Suspected change region

Definition 2(behavioural profile of Petri net). Let $N=$ $(S, T ; F)$ is a Petri net. A pair of transitions $(x, y) \in(T \times T)$ can be in the following profile relations:

1.The strict order relation $\rightarrow_{P}$, if $x \succ_{P}$ y and $y \nsucc_{P} x$;

2.The exclusiveness relation $+_{P}$, if $x \nsucc_{P} y$ and $y \nsucc_{P} x$;

3.The interleaving order relation $\|_{p}$, if $x \succ_{P} y$ and $y \succ_{P} x$.

The set $B_{P}=\left\{\rightarrow_{P},+_{P}, \|_{P}\right\}$ is the behavioural profile of $N=(S, T ; F)$.

Due to the special relationship between a pair of transitions $(x, y) \in(T \times T)$, this paper gives the notion of the order relations graph.

Definition 3(Order relations graph). Let $B_{P}=\left\{\rightarrow_{P},+P\right.$, $\left.\|_{P}\right\}$ is the behavioural profile of Petri net $N=(S, T ; F)$. A tuple $r=(T, E)$ is the order relations graph of $N$, if it satisfies the following conditions:

1.T is the set of node, that is the set of all transitions in $N=(S, T ; F)$;

2.E is the set of edge, corresponding to the order relation between nodes.

For instance, Fig. 4 is an order relations graph of Fig. 3. It clearly illustrates the order relation between transitions. Further more, to obtain more accurate change region of process model, this paper gives the notion of change region.

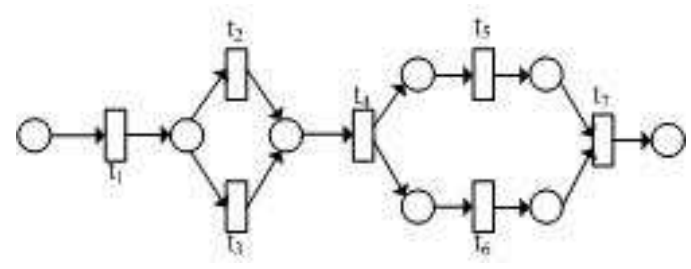

Fig. 3: Petri net model

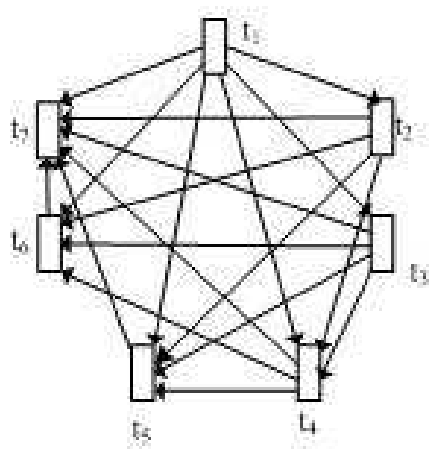

Fig. 4: The order relations graph of Petri net

Definition 4(Change region). Let $N=(S, T ; F)$ is Petri net. If change region is $N^{1}=\left(S^{1}, T^{1} ; F^{1}\right), N^{1}$ is a subnet of $N$, and $S^{1} \in S, T^{1} \in T, F^{1} \in F, N^{1} \subseteq N$.

If for any $N^{2} \subseteq N^{1}$, there does not exist $N^{3}$, holding $\left|S^{3}\right|<\left|S^{2}\right|,\left|T^{3}\right|<\left|T^{2}\right|, N^{2}$ is the minimal change region of $N$. So change region of Petri net model is the subnet of this net model, and its places and transitions in minimal change region are covered by places and transitions in change region.

On the basis of these notions, this paper adopts the definitions of Petri net and its behavioural profile, presenting a new method to study change region of process model.

\section{Identifying change region of business process model based on Petri net}

\subsection{Background}

Modular decomposition theory stems from the research of Gallai on transmitting direction [11]. This method is often applied in the diagram, 2-structure, classification and so on, in $[12,13,14,15]$. With the development of technology, this way is commonly used in process model. For example, in constructing the abstract model of business process model, [9] presents a way to build it after using modular decomposition of the original business process model. Visibly, decomposition of process model is not only clearly showing the relationship 
of pairs of activities, but also can get process model by combination of module.

Because modular decomposition can refine the process model, there exist behavioural relations in internal module and between modules. This paper firstly presents a method of modular decomposition and then analyzes the behavioural relations in modules to identify the change region. Its advantages are that avoid the shackles of the target model, and get more accurate change region with increasing accuracy.

Consequently, combining with the advantages of modular decomposition, this section starts with introducing the elementary notions, and then analyzes the behavioural relationships between modules. Finally, change region is obtained by using this way.

Definition 5(modular decomposition). Let $r=(T, E)$ is an order relation graph.

1.M is a module, if $M \neq \emptyset, M \subseteq T$, then $\forall x, y \in M, z \in$ $(T / M)$, it holds

$$
(x, z) \in E \Leftrightarrow(y, z) \in E
$$

and

$$
(x, z) \in E \Leftrightarrow(z, y) \in E
$$

2.M is a trivial module, if it meets $M=\emptyset$ or $M=T$ or $M=\{t\} \in T$.

3.M is a simple module, if there does not exist $M_{1} \subseteq T$, holding $M_{1} \subseteq M$.

Modular decomposition is a tuple $M G_{r}=(\Omega, X)$, such that $\Omega$ is a set of all simple modules and $X: \Omega \rightarrow \rho(\Omega)$ is a function.

According to this definition, there exist relations between modules. So the definition of behavioural profile of module is given.

Definition 6(behavioural profile of module). Let $N=(S, T ; F)$ is Petri net, and its set of non-trivial modules is $\Omega$. A pair of modules $(M, N) \in(Q \times Q)$ can be in the following profile relations:

1.The order relation $\Rightarrow_{P}$, such that $\forall x \in M, y \in N$ it holds $(x, y) \in E$ and $(y, x) \notin E$.

2.The parallel relation $\bigoplus_{P}$, such that $\forall x \in M, y \in N$, it holds $(x, y) \notin E$ and $(y, x) \notin E$.

3.The circular relation $\bigotimes_{P}$, such that $\forall x \in M, y \in N$, it holds $(x, y) \in E$ and $(y, x) \in E$.

The set of $M B_{P}=\left\{\Rightarrow_{P}, \bigoplus_{P}, \bigotimes_{P}\right\}$ is behavioural profile of module of $N=(S, T ; F)$.

In Fig. 5, $M_{1}, M_{2}$ and $M_{3}$ is the order relation. $M_{1}$ and $M_{2}$ is the parallel relation. In addition, the circular relation of module is performed between $M_{1}$ and $M_{2}$ in Fig. 6 .

To better reflect the change region of modules, change module is introduced.

Definition 7(change module). Let $N=(S, T ; F)$ is Petri net and the set of simple modules is $\Omega$. If a change region is the module $M_{1}, M_{1} \in \Omega$ is a change module.

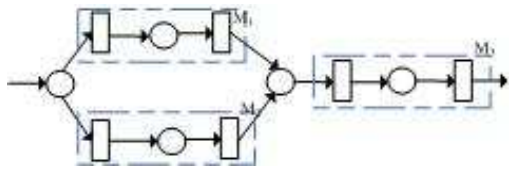

Fig. 5: The order relation and the parallel relation of module

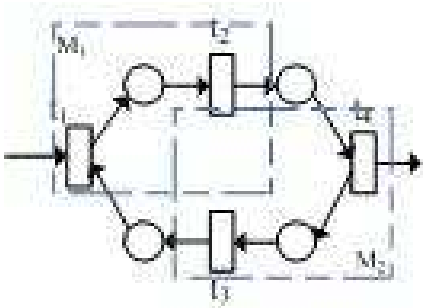

Fig. 6: The circular relation of module

This paper analyzes behavioural profile of modules to seek the change region of process model. Based on the notions of Petri net and behavioural profile, it begins with modular decomposition of models, and then gets the behavioural profile of modules by analyzing the behavioural relations of modules. Finally, according to the characteristics and the behavioural profile of the module, it can accurately identify the minimal change region by observing different input and output transitions and the point of dynamic analysis. This method avoids dependence on the target model, and improves the limitation of static analysis.

\subsection{Algorithm}

For identifying the minimal change region, this section gives two algorithms.

Algorithm 1: locate the change region based on module.

Input: a Petri net $N=(S, T ; F)$ with its first transition $t_{0}$ and its terminal transition $t_{N}, N=|T|$, its behavioural profile

$$
B_{P}=\left\{\rightarrow_{P},+_{P}, \|_{P}\right\}
$$

its order relation graph $r=(T, E)$ with the set of all non-trivial simple modules $\Omega$, its behavioural profile of module $M B_{P}=\left\{\Rightarrow_{P}, \bigoplus_{P}, \bigotimes_{P}\right\}$. With $M_{1}, M_{2} \in \Omega$, the set of normal modules $M_{n}$ and the set of change modules $M_{r}$, its relational function $\tau:\left(M_{1}, M_{2}\right) \rightarrow \tau\left(M_{1}, M_{2}\right)$.

Output: $M_{r}$, the set of suspected transitions $T_{0}$ and change region $W$. 
(1) Choosing $t_{N}$ as the observing node, if $N=(S, T ; F)$ can run to the end, returns to the step (2); otherwise, exits the system.

(2) According to definition 3, the order relation graph $r=(T, E)$ is given, and then obtains $\Omega$ by the partitions of $N=(S, T ; F)$ based on modular decomposition according to definition 5 .

(3) Transitions $t_{1}, t_{2}, t_{3}, t_{4}, \ldots, t_{i-1}, t_{i}$ are the order of all commonly output transitions between modules.

(4) In $N=(S, T ; F)$, choosing sequentially the output transition $t_{m}$ is the observing point, and then finds the set $M(m=0,1,2, \ldots, i-1)$ of modules who are not marked with locating $t_{m}$ as the input transition. According to definition 6, there analyzes the relations of all pairs of modules $\left(M_{1}, M_{2}\right)$.

(5) If $\forall x \in M_{1}, y \in M_{2}$

1) then $(x, y) \in E$ and $(y, x) \notin E$, it holds $\tau\left(M_{1}, M_{2}\right)=\left\{\Rightarrow_{P}\right\} \in M B_{P}$, returns to the step (6); otherwise, returns to (7).

2) then $(x, y) \notin E$ and $(y, x) \notin E$, it holds $\tau\left(M_{1}, M_{2}\right)=\left\{\bigoplus_{P}\right\} \in M B_{P}$, returns to the step (6); otherwise, returns to (7).

3) then $(x, y) \in E$ and $(y, x) \in E$, it holds $\tau\left(M_{1}, M_{2}\right)=\left\{\bigotimes_{P}\right\} \in M B_{P}$ returns to the step (6); otherwise, returns to (7).

(6) Until to the end of the system, puts $M_{1}$ and $M_{2}$ into $M_{n}$ and then is numbered sequentially; otherwise, returns to the step (4).

(7) According to definition 7, until to the end of the system, puts $M_{1}$ and $M_{2}$ into $M_{r}$ and then is numbered sequentially; otherwise, returns to the step (4).

(8) Finally, it can obtain a set of change modules $M_{r}=\left\{M_{1}, M_{2}, \ldots, M_{s}\right\}$, a set of suspected transitions $T_{0}=M_{1} \cup M_{2} \cup \ldots, \cup M_{s}$, and the corresponding change region $W=T_{0} \cup^{\bullet} T_{0} \cup T_{0}^{\bullet}$.

Algorithm 2: identify the minimal change region

Input: the change region $W$, a set of suspected transitions and a net $G=\left(N, M_{0}\right)$ with initial marking $M_{0}$.

Output: the minimal change region $N_{0}$.

(1) According to algorithm 1, the set of change modules in $N=(S, T ; F)$ is given, and then gets a net $G=\left(N, M_{0}\right)$ with an initial marking $M_{0}$.

(2) Let lists all occurring sequences passing the change region and in the set of suspected transitions $T_{0}: \sigma_{1}, \sigma_{2}, \ldots, \sigma_{s}, 1 \leq i \leq s$.

(3) Selecting a sequence $\sigma_{i}$, for any two places $s_{i}$ and $s_{j}$ with $s_{i} \neq s_{j}$ and $i<j$ in $T_{0} \cap \sigma_{i}$,

1)If $\bullet s_{i} \neq \emptyset$ or $\bullet s_{j} \neq \emptyset$, it holds

$$
G_{1}=s_{i} \cup \bullet s_{i} \cup s_{j} \cup \bullet s_{j} \cup \ldots
$$

triggered by it, until to the end of the system; otherwise, holds $G_{1}=\sigma_{i}$.
2)If $s_{i}^{\bullet} \neq \emptyset$ or $s_{j}^{\bullet} \neq \emptyset$, it holds

$$
G_{2}=s_{i} \cup s_{i}^{\bullet} \cup s_{j} \cup s_{j}^{\bullet} \cup \ldots
$$

launching backward enabled transitions and places triggered by it, until to the end of the system; otherwise, holds $G_{2}=\sigma_{i}$.

3)Let notes change region to be

$$
W_{i}=G_{1} \cap G_{2} \cap W .
$$

(4) Let returns to the step (2), operating every sequence.

(5) Let gives the minimal change region $N_{0}=\bigcup_{i=1}^{m} W_{i}$.

\subsection{Example analysis}

For the existing methods, they need to depend on the complex and inaccurate target model, and even require to analyze the fixed observing point, such that it is difficult to seek accurate the change region of process model. To solve these problems, this paper analyzes the behavior of modules. This method avoids dependence on the target model, and gets more accurate change region of process model by dynamic analysis.

For the above example of Fig. 1, algorithm 1 analyzes the consistency between the behavioural relations of modules and the definition of its behavioural profile, so the dashed part in Fig. 7 shows $T_{0}$ and the change region with a dotted border. Then, according to algorithm 2, all occurring sequences in the change region is "Pay for the order, Finish the order, Transaction success", "Pay for the order, Payment, Payment success, Transaction success", and "Pay for the order, Modify the order, Refund, Payment, Payment success, Transaction success". Examining these sequences, the place in the dashed part is used for the condition. Then it launches forward and backward respectively to get enabled transitions and triggered places. The rest may be deduced by analogy and gets $G_{1}$ and $G_{2}$ in Fig. 8. Finally, the result is

$$
W_{1}=G_{1} \cap G_{2}=\{\text { payment success }\}
$$

Based on this way, after analyzing others, the ultimate change region is $\{$ Payment success, Transaction success $\}$.

\section{Conclusion}

According to the advantages and disadvantages of existing researches on the change region of process models, this paper expects to present a method which can rapidly and accurately seek the change region. And then modular decomposition is introduced based on the definition of order relation graph and module. Furthermore, the definition of behavioural relations of module is given. Finally, this paper presents a novel 


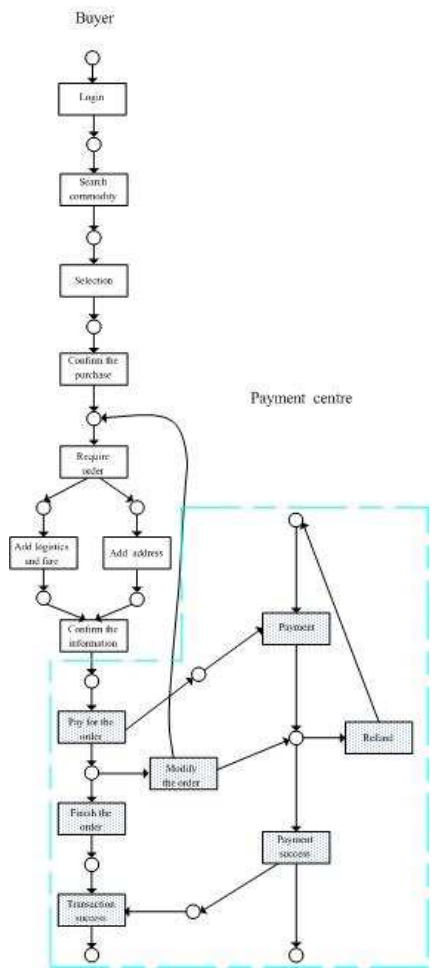

Fig. 7: The set of $T_{0}$ and the change region $W$

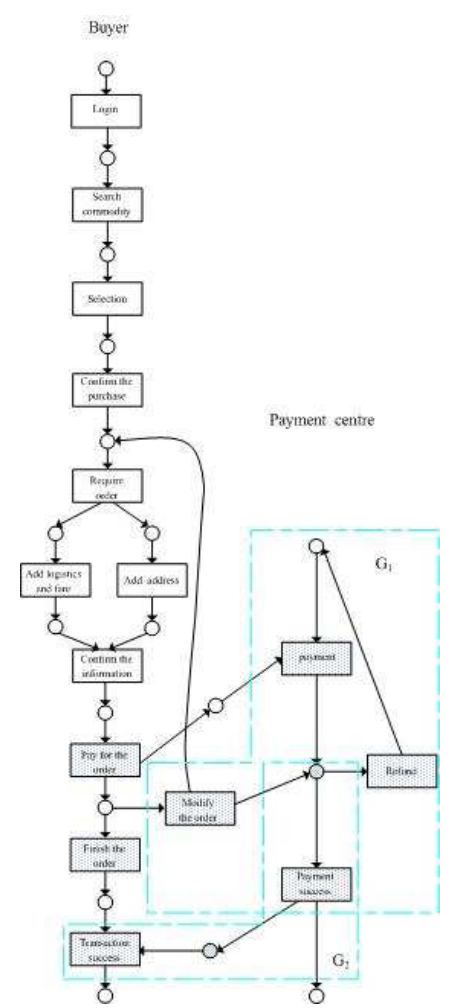

Fig. 8: Change region $W_{1}$ method to identify the change region, before analyzes the example.

The contributions of this paper are that (1) analyzes the model itself without the target model, for seeking the change region; (2) according to existing knowledge, the behavioural profile of module is defined, expanding the scope of application of behavioural profile, such that it provides the standard of judging the change modules; (3) due to the uncertain of static analysis, this paper observes different input and output transitions to seek the accurate change region by dynamic operation.

By analyzing the behavioural relations of modules, this paper identifies the change region of process model, however, the implementing case of adding token is not considered. This method has limitation.

In future, further work is also studied on the application of behavioural profile of module to seek the change region of process model. For example, the problem of how to apply modular behaviors to analyze the behavioural consistency of internal modules and external modules for identifying the change region is remained to be solved.

\section{Acknowledgement}

We would like to thank the support of the National Natural Science Foundation of China under Grant No.61272153, No.61100058, No.60973050, No.61170059 and No.61170172, the Natural Science Foundation of Educational Government of Anhui Province of China ( KJ2012A073, KJ2011A086), Anhui Provincial Natural Science Foundation (1208085MF105), Anhui Provincial Soft Science Foundation (12020503031).

\section{References}

[1] C. Gerth, M. Luckey, J. M. Kster, G. Engels. Detection of Semantically Equivalent Fragments for Business Process Model Change Management. In 2010 IEEE International Conference on Services Computing. 56-64 (2010)

[2] Monika Weidmann, Modood Alvi, Falko Koetter, Frank Leymann, Thomas Renner, David Schumm. Business Process Change Management based on Process Model Synchronization of Multiple Abstraction Levels. In 2011 IEEE International Conference on Service-Oriented Computing and Applications. 1-4 (2011).

[3] Matthias Weidlich, Jan Mendling, Mathias Weske. Propagating changes between aligned process models. In Journal of Systems and Software, 85, 1885-1898 (2012).

[4] Matthias Weidlich, Mathias Weske, Jan Mendling. Change Propagation in Process Models using Behavioural Profiles. In 2009 IEEE International Conference on Services Computing, 33-40 (2009)

[5] Egyed A. Fixing inconsistencies in UML models. In proceedings of the 29th international conference on software engineering, 29, 292-301 (2007). 
[6] Dam Hoa Khanh, Winikoff Michael. An agent-oriented approach to change propagation in software maintenance. Autonomous Agents and Multi-Agent Systems, 23, 384-452 (2011).

[7] Fei Genyuan, Gao James, Owodunni Oladele. A method for engineering design change analysis using system modeling and knowledge management techniques. Computer Integrated, 24, 535-551 (2011).

[8] Remco M. Dijkman. Diagnosing differences between business process models. BPM '08 Proceedings of the 6th International Conference on Business Process Management, 5240, 261-277 (2008).

[9] Jan Mendling. Business Process Model Abstraction Based On Synthesis From Well-structured Behavioral Profiles. International journal of cooperative information systems, 21, 55-83 (2012).

[10] Zhehui Wu. Petri net theory. China Machine Press, 6-42 (2006).

[11] T. Gallai. Transitiv Orientierbare Graphen. Acta Mathematica Hungarica - ACTA MATH HUNG, 18, 25-66 (1967).

[12] Alain Cournier, Michel Habib. A New Linear Algorithm for Modular Decomposition. Lecture Notes in Computer Science, 787, 68-84 (1994).

[13] Ross M. McConnell, Fabiende Montgolfier. Liner-time modular decomposition of directed graphs. Discrete Applied Mathematics, 145, 198-209 (2005).

[14] Matthias Weidlich, Artem Polyvyanyy, Jan Mendling, MathiasWeske. Efficient and Practical Modular Decomposition. Proceeding SODA '97 Proceedings of the eighth annual ACM-SIAM symposium on Discrete algorithms, 26-35 (1997).

[15] R. H. MShring, F. J. Radermacher. Substitution decomposition for discrete structures and connections with combinatorial optimization. Ann Discrete math., 19, 257-356 (1984).

[16] Matthias Weidlich, Jan Mendling. Perceived consistency between process models. Information Systems, 37, 80-98 (2012).

[17] Eramo Romina, Malavolta Ivano, Muccini Henry, et al. A model-driven approach to automate the propagation of changes among Architecture Description Languages. Software and Systems Modeling. 11, 29-53 (2012).

[18] Jussi Vanhatalo, Hagen Völzer, Jana Koehler. The refined process structure tree. Data Knowledge, 68, 793-818 (2009).

[19] Jochen Malte Küster, Christian Gerth, Alexander Förster, Gregor Engels. Detecting and resolving process model differences in the absence of a change log. BPM ' 08 Proceedings of the 6th International Conference on Business Process Management, 244-260 (2008).

[20] Matthias W, Artem P, Nirmit D, et al. Process compliance analysis based on behavioral profiles[J]. Information Systems, 36, 1009-1025 (2011).

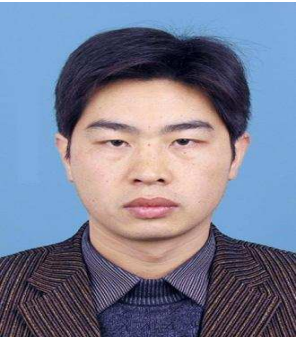

Xianwen Fang received his M.A. degree from Shandong University of Science and Technology, China, in 2004, and PhD. degree in the key Lab of Service Computing at Tongji University in 2011. He is currently a Professor with the Department of Computer Science and Engineering, Anhui University of Science and Technology, China. His research interests include Petri net, trustworthy software and Web services. $\mathrm{He}$ has published more than 60 papers in domestic and international academic journals and conference proceedings. These papers are embodied more than 40 times by SCI and EI and are cited more than 100 times by others.

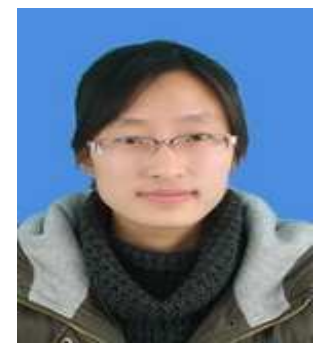

Lu Liu received the M.A. degree from Anhui University of Science and Technology, China, in 2012. She is currently a lecturer with the Department of Computer Science and Engineering, Anhui University of Science and Technology, China. Her current areas of research are concurrent theory, Petri net and formal verification of software.

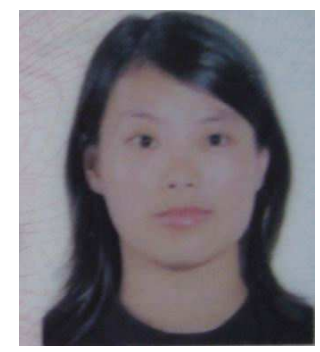

Xiangwei Liu received the M.A. degree from Anhui University of Finance and Economics, China, in 2005. She is currently an associate professor with the Department of Computer Science and Engineering, Anhui University of Science and Technology, China. Her current areas of research are Web service computing, Petri net and formal verification of software. He has published more than 20 papers in the international academic journals, these papers are embodied more than 20 times by SCI and EI and are cited more than 60 times by others. 